\title{
Field Plate Design for Low Leakage Current in Lateral GaN Power Schottky Diodes: Role of the Pinch-off Voltage
}

\author{
Jun Ma, Dante Colao Zanuz, and Elison Matioli, Member, IEEE
}

\begin{abstract}
In this letter, we demonstrate a general model to reduce the reverse leakage current $\left(I_{R}\right)$ in high-voltage AIGaN/GaN Schottky diodes (SBDs) by engineering the pinchoff voltage $\left(V_{p}\right)$ of their field plates (FPs). The maximum voltage drop at the Schottky junction $\left(V_{\mathrm{SCH}}\right)$ in the off state can be significantly decreased by reducing $\left|V_{\mathrm{p}}\right|$, which leads to a drastically diminished $I_{R}$. We used a tri-gate architecture as means to control $V_{p}$ and, thus, $I_{R}$, as it offers great flexibility to engineer $V_{\mathrm{p}}$ compared with conventional schemes. $\left|V_{\mathrm{p}}\right|$ of SBDs with tri-gate FPs was reduced by decreasing the width of the nanowires, which led to a very small $I_{R}$, below $10 \mathrm{nA} / \mathrm{mm}$ under reverse biases up to $500 \mathrm{~V}$, and an increase of over $800 \mathrm{~V}$ in soft breakdown voltage $\left(V_{\mathrm{BR}}\right)$ at $1 \mu \mathrm{A} / \mathrm{mm}$. These results reveal the importance of $V_{p}$ in reducing $I_{R}$ for SBDs, and unveil the potential of tri-gate structures as FPs for power devices.
\end{abstract}

Index Terms-GaN, field plate, Schottky diode, breakdown, leakage current, tri-gate.

\section{INTRODUCTION}

$\mathbf{L}$ ATERAL AlGaN/GaN SBDs are very promising for power conversions, offering excellent properties for high-voltage, high-power-density and high-frequency operation [1]-[10]. In addition, these devices can be monolithically integrated with GaN high electron mobility transistors (HEMTs) on large-size silicon substrates, which is highly desirable to reduce the cost, size and parasitics for future $\mathrm{GaN}$ power converters.

A major obstacle for current lateral $\mathrm{AlGaN} / \mathrm{GaN}$ SBDs is however their large $I_{\mathrm{R}}$. Efficient and reliable power conversion requires devices with small $I_{\mathrm{R}}$ below $1 \mu \mathrm{A} / \mathrm{mm}$ or preferably $0.1 \mu \mathrm{A} / \mathrm{mm}$ at high blocking voltages [11], yet most of current SBDs exhibit $I_{\mathrm{R}}$ over $0.1 \mu \mathrm{A} / \mathrm{mm}$ at a reverse bias as small as $100 \mathrm{~V}$, leading to a small $V_{\mathrm{BR}}$ and a large power dissipation in OFF state. While many sophisticated techniques have been

Manuscript received June 30, 2017; revised July 19, 2017; accepted July 25, 2017. Date of publication July 31, 2017; date of current version August 23, 2017. This work was supported in part by the European Research Council under the European Union's H2020 Program/ERC grant Agreement 679425 and in part by the Swiss National Science Foundation under Assistant Professor Energy Grant PYAPP2_166901. The review of this letter was arranged by Editor D. G. Senesky. (Corresponding authors: Jun Ma; Elison Matioli.)

The authors are with the Power and Wide-band-gap Electronics Research Laboratory, École polytechnique fédérale de Lausanne, $\mathrm{CH}-1015$ Lausanne, Switzerland (e-mail: jun.ma@epfl.ch; elison.matioli@epfl.ch).

Color versions of one or more of the figures in this letter are available online at http://ieeexplore.ieee.org.

Digital Object Identifier 10.1109/LED.2017.2734644 proposed to address this issue, their effect is limited by parasitic leakage paths under high biases, such as thermionic field emission and trap-assisted tunneling [12]-[19].

Recently we have demonstrated tri-anode $\mathrm{AlGaN} / \mathrm{GaN}$ SBDs with small $I_{\mathrm{R}}(\leq 0.1 \mu \mathrm{A} / \mathrm{mm}$ at $-700 \mathrm{~V})$ [1]. Small $I_{\mathrm{R}}$ was also reported by $\mathrm{Hu}$ et al. [7] using a gated-edge termination, in which the $\mathrm{AlGaN}$ barrier in the FP was partially recessed. The $I_{R}$ in these reports are comparable to state-ofthe-art GaN transistors and much smaller than other results from lateral $\mathrm{AlGaN} / \mathrm{GaN}$ SBDs in the literature [2]-[5]. Yet the physical origin of such improvement is not well understood. More importantly, a general model for the reduction of $I_{\mathrm{R}}$ is still missing, which is crucial to unleash the full potential of lateral SBDs for the next generation of power converters.

In this work we present a general approach to reduce the $I_{\mathrm{R}}$ in SBDs by designing the $V_{\mathrm{p}}$ of their FPs, which, in addition to explaining the improvement mentioned above, provides a pathway for high-performance lateral GaN power Schottky diodes. A reduction of $\left|V_{\mathrm{p}}\right|$ results in a decreased $V_{\mathrm{SCH}}$ in OFF state and correspondingly a smaller $I_{\mathrm{R}}$. To verify the model, we used a tri-gate structure to reduce the $\left|V_{\mathrm{p}}\right|$ in $\mathrm{AlGaN} / \mathrm{GaN}$ SBDs, which resulted in very small $I_{\mathrm{R}}$, below $10 \mathrm{nA} / \mathrm{mm}$ at $-500 \mathrm{~V}$, along with an enhancement over $800 \mathrm{~V}$ in $V_{\mathrm{BR}}$.

\section{Model And Methodology}

The $I_{\mathrm{R}}$ in SBDs is determined by three components: 1. Leakage by thermionic emission, which comprises the $I_{R}$ of an ideal SBD; 2. Thermionic field emission, tunneling and other similar non-ideal effects $\left(I_{\mathrm{FE}, \mathrm{T}}\right)$, which dominate the $I_{\mathrm{R}}$ in real devices [12]-[19]; 3. Leakage through buffer layers, which is negligible under low voltages for SBDs on highresistivity buffer layers. Many sophisticated schemes have been proposed to reduce the $I_{\mathrm{FE}, \mathrm{T}}$ by reducing defects and traps [20]-[23], yet the $I_{\mathrm{R}}$ is still large in most of GaN-onsilicon SBDs. This is likely due to the high electric field under large reverse biases and also the high defect density in GaN on silicon [24]-[26].

However, $I_{\mathrm{FE}, \mathrm{T}}$ is not only determined by the density and energy levels of the traps, but also increases with the voltage drop at the Schottky junction $\left(V_{\mathrm{SCH}}\right)$ (or the electric field $\left.\left(E_{\mathrm{SCH}}\right)\right)$. In this work we propose an approach to reduce the $V_{\mathrm{SCH}}$ and $E_{\mathrm{SCH}}$ by reducing the $\left|V_{\mathrm{p}}\right|$ of the FPs, which in addition to reducing the $I_{\mathrm{R}}$ also increases the $V_{\mathrm{BR}}$ of the SBDs. To illustrate the principle, we simulated the distribution of potential $(\Phi)$ and electric field $(E)$ in a typical SBD

(c) 2017 IEEE. Personal use of this material is permitted. Permission from IEEE must be obtained for all other uses, in any current or future media, including reprinting/republishing this material for advertising or promotional purposes, creating new collective works, for resale or redistribution to servers or lists, or reuse of any copyrighted component of this work in other works. 

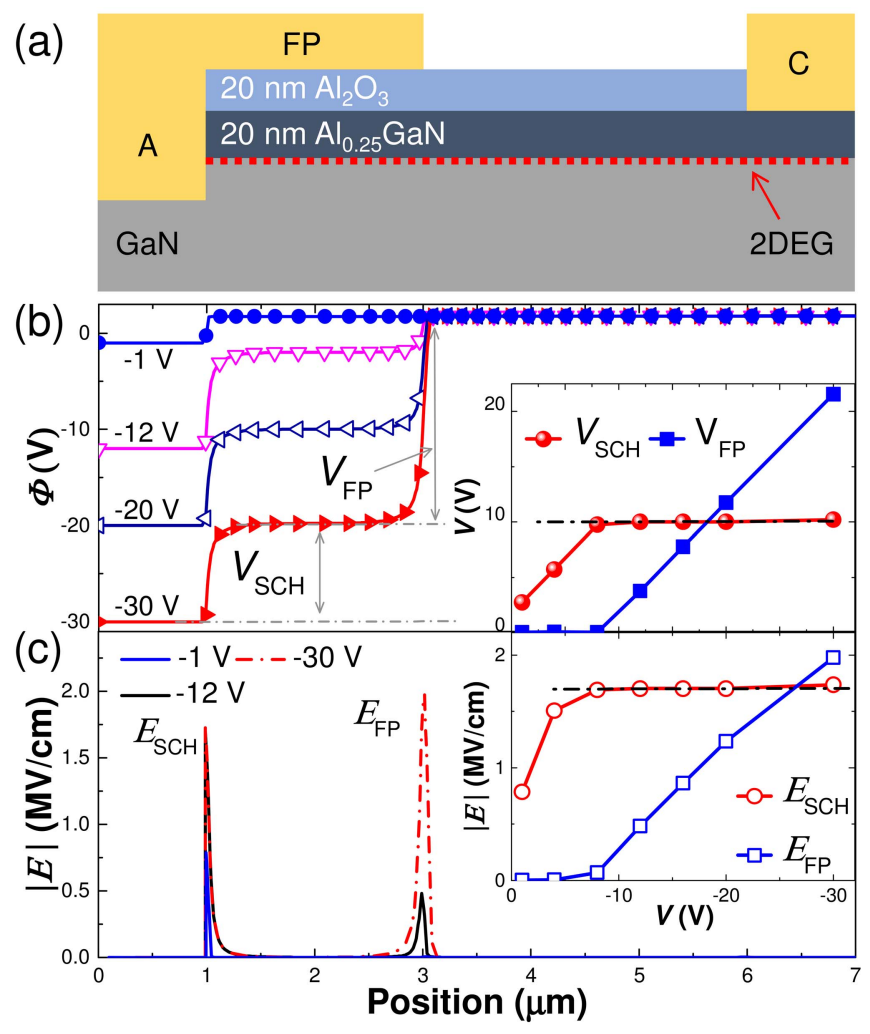

Fig. 1. (a) A cross-sectional schematic of a lateral AIGaN/GaN SBD with a typical planar FP. Simulated distributions of $(b)$ potential $(\Phi)$ and (c) electric field $(E)$ at the channel in OFF state for different anode voltages, in which only in-plane electric field was considered. The insets show the summarized dependences of the $V_{\mathrm{SCH}}$ and the $E_{\mathrm{SCH}}$ on the anode voltage.

with a recessed anode and a conventional planar FP in OFF state (Fig. 1), using Atlas Silvaco. When the reverse bias in the anode is smaller than $\left|V_{\mathrm{p}}\right|$, which in this case is about $10 \mathrm{~V}$ (extracted by simulating a transistor with the FP as the gate), the voltage drops only at the Schottky junction (inset of Fig. 1(b)). As the reverse bias reaches $\left|V_{\mathrm{p}}\right|$, the FP depletes the channel beneath and the voltage starts to drop at the cathodeside edge of the FP, resulting in a second peak of electric field. Then $V_{\mathrm{SCH}}$ saturates at $\left|V_{\mathrm{p}}\right|$, regardless of further increase of the bias, as summarized in the insets of Figs. 1(b) and (c), respectively. These results agree well with [27] and [28], and suggest that the FP can be used to control the $I_{\mathrm{R}}$ in SBDs, as a smaller $\left|V_{\mathrm{p}}\right|$ reduces the maximum $V_{\mathrm{SCH}}$ and $E_{\mathrm{SCH}}$ at the Schottky junction and hence diminishes the $I_{R}$.

To reduce the $\left|V_{\mathrm{p}}\right|$, a few ways can be adopted, including a partial recess of the $\mathrm{AlGaN}$ barrier layer. However, a precise control to obtain a series of $V_{p}$ with these methods is very challenging, which makes them less suited to explore the $\left|V_{\mathrm{p}}\right|$ dependence of the $I_{\mathrm{R}}$. Here we used a tri-gate structure to reduce the $\left|V_{\mathrm{p}}\right|$ [29], [30], as it offers great control to tune the $V_{\mathrm{p}}$ by changing the width of the nanowires $(w)$ in the tri-gate. We implemented tri-gate FPs in SBDs for both conventional recessed anodes and novel tri-anodes [1] to justify our approach, and compared them with other literature results to show the generality of the model.

The fabrication of all SBDs in this work started with e-beam lithography to define the nanowires and the mesa, which were

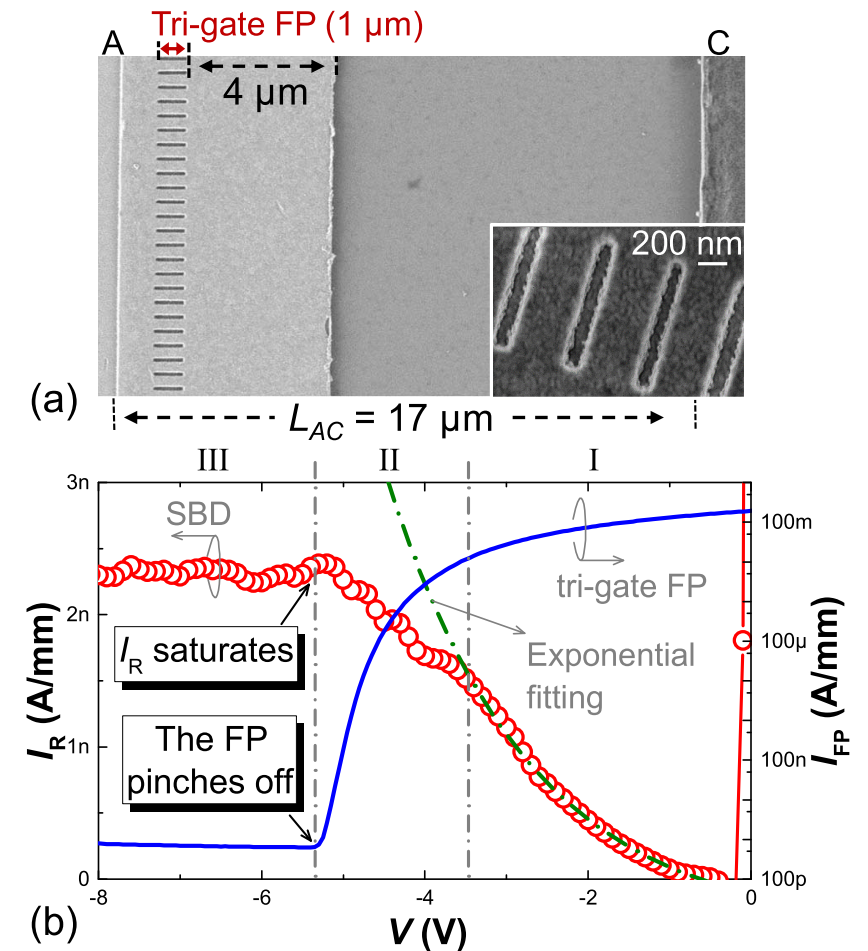

Fig. 2. (a) Top-view SEM images of the fabricated AIGaN/GaN SBD with a tri-gate FP. (b) $I_{R}$ of the SBDs and the pinch-off characteristic of the tri-gate FPs as a function of the voltage. All characteristics were averaged from about 8 devices of the same kind and normalized by their total width of $60 \mu \mathrm{m}$. The turn-ON voltage $\left(V_{\mathrm{ON}}\right)$ of these SBDs was $0.9 \pm 0.1 \mathrm{~V}$, extracted at a forward current of $1 \mathrm{~mA} / \mathrm{mm}$.

later etched using inductively coupled plasma with a depth of $\sim 160 \mathrm{~nm}$, followed by the formation of ohmic contacts in the cathode region. Then $20 \mathrm{~nm}$ of $\mathrm{Al}_{2} \mathrm{O}_{3}$ was deposited by atomic layer deposition as the FP oxide, which was selectively removed in part of the anode region to form the Schottky contact. Finally Ni/Au was deposited as the anode and then used as the mask to remove the oxide in access regions by wet etching. All SBDs shared similar device dimensions such as a cathode-to-anode separation $\left(L_{\mathrm{AC}}\right)$ of $17 \mu \mathrm{m}$ and a total device width of $60 \mu \mathrm{m}$.

\section{Results And Discussion}

In the model presented in Section II, the $I_{\mathrm{R}}$ should saturate when the $V_{\mathrm{SCH}}$ is pinned after the pinch-off of the FP. To verify this, we compared the $I_{R}$ of an SBD and the pinch-off characteristics of its FP (Fig. 2). The SBD had $1 \mu \mathrm{m}$-long tri-gate FPs (Fig. 2(a)), in which the $w$ and the spacing of the nanowires were $300 \mathrm{~nm}$ and $200 \mathrm{~nm}$, respectively. These SBDs had recessed anodes with a recess depth of $\sim 160 \mathrm{~nm}$. The average pinch-off characteristic of the tri-gate FPs, shown in Fig. 2(b), was determined from transfer characteristics of tri-gate GaN MOSHEMTs on the same chip at $V_{\mathrm{DS}}$ of $5 \mathrm{~V}$. The nanowires in the MOSHEMTs had a $w$ of $300 \mathrm{~nm}$ with a length of $700 \mathrm{~nm}$. As shown in Fig. 2(b), $I_{\mathrm{R}}$ increases exponentially with the reverse bias in Region I, which is dominated by $I_{\mathrm{FE}, \mathrm{T}}$. As the FP starts to pinch off the channel, the increase of $I_{\mathrm{R}}$ slows down (Region II). Finally $I_{\mathrm{R}}$ saturates as the FP completely pinches off the channel (Region III), agreeing well with our model. 

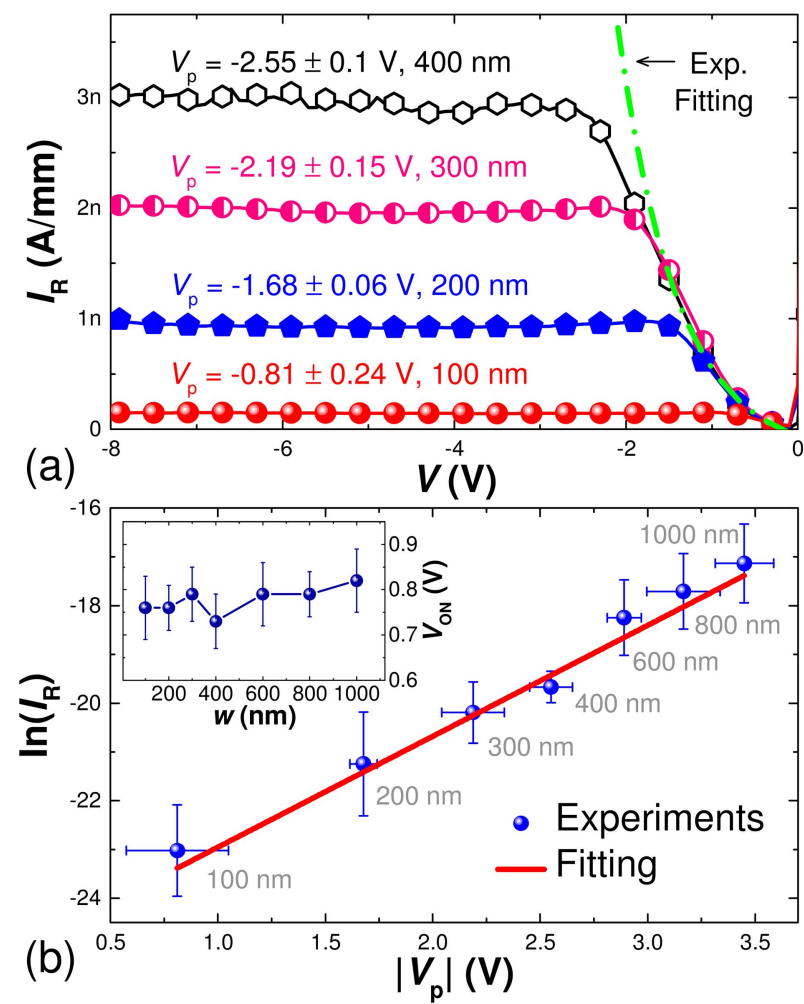

Fig. 3. (a) Comparison of the $I_{R}$ in hybrid tri-anode SBDs with different $w$ and thus different $V_{\mathrm{p}}$. (b) A linear fitting of the $\ln \left(I_{\mathrm{R}}\right)$ at $-10 \mathrm{~V}$ and the $\left|V_{\mathrm{p}}\right|$. The inset shows the $V_{\mathrm{ON}}$ of these devices as a function of $w$. The spacing between nanowires in these devices was $200 \mathrm{~nm}$. $l_{R}$ was normalized by their total width of $60 \mu \mathrm{m}$ (the width of the device footprint) and the error bars were determined from about 8 devices of each kind.

To demonstrate the dependence of the $I_{\mathrm{R}}$ with the $\left|V_{\mathrm{p}}\right|$, we studied tri-anode SBDs [1], since their $\left|V_{\mathrm{p}}\right|$ can be reduced by decreasing the $w$ of the tri-anode (which are basically trigate HEMTs without the oxide). Figure 3(a) shows the $I_{\mathrm{R}}$ of tri-anode $\mathrm{AlGaN} / \mathrm{GaN}$ SBDs with different $w$, thus different $V_{\mathrm{p}}$, which follow a similar exponential increase before the pinch off of their FPs and then saturate as $V$ reaches $V_{\mathrm{p}}$. As $\left|V_{\mathrm{p}}\right|$ decreases for narrower nanowires, the saturated $I_{\mathrm{R}}$ diminishes due to the reduced $V_{\mathrm{SCH}}$. This reduction of $I_{\mathrm{R}}$ is not likely caused by the change of the Schottky barrier height, as we observed little dependence of the $V_{\mathrm{ON}}$ on the $w$ (the inset of Fig. 3(b)). Figure 3(b) shows a linear relationship of the $\ln \left(I_{\mathrm{R}}\right)$ with $\left|V_{\mathrm{p}}\right|$, due to the exponential increase of $I_{\mathrm{R}}$ before the pinch off of the FPs (Fig. 3(a)). Such linear dependence was not affected by the choice of normalization of the $I_{R}$ (either by device width, effective device width or number of nanowires).

The reduction of $I_{\mathrm{R}}$ with $\left|V_{\mathrm{p}}\right|$ can be generally applicable to devices with different approaches to tune the pinch-off voltage, not only to tri-anode SBDs. For instance, a reduced $\left|V_{\mathrm{p}}\right|$ was achieved by recessing the AlGaN barrier in the FP [7] or by thinner FP oxide [31], both of which led to a significant reduction of $I_{\mathrm{R}}$. The $I_{\mathrm{R}}$ from state-of-the-art AlGaN/ GaN-on-silicon SBDs with different FP designs were compared in Fig. 4. SBDs with no FPs or conventional FPs (grey) exhibited large $I_{\mathrm{R}}$ beyond $0.1 \mu \mathrm{A} / \mathrm{mm}$ at very small biases, while the $I_{\mathrm{R}}$ was much smaller in SBDs with reduced $\left|V_{\mathrm{p}}\right|$ by either AlGaN recess (blue) or tri-gate FPs (orange). This observation from different groups in the literature supports

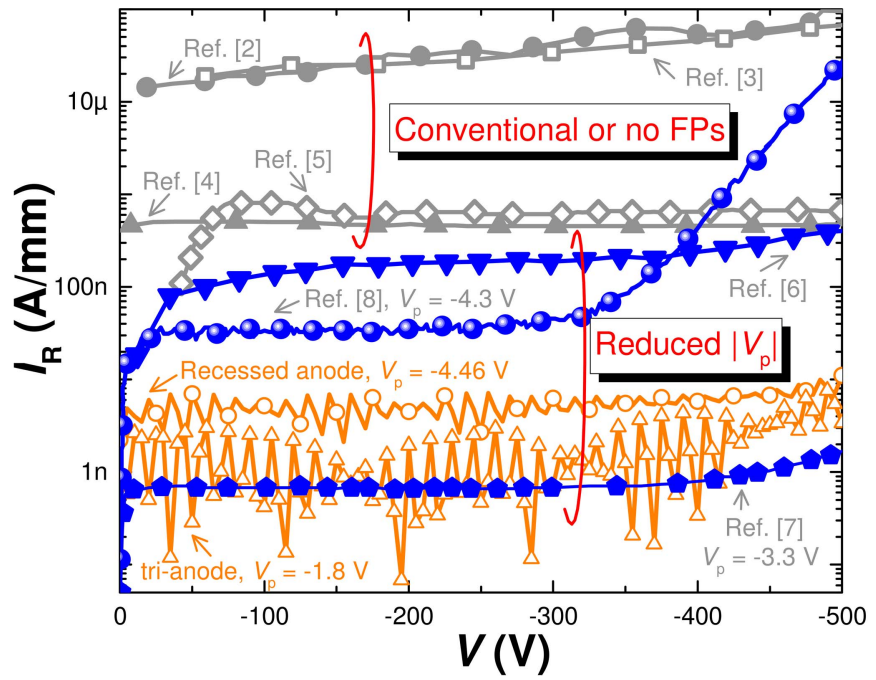

Fig. 4. Comparison of $I_{R}$ from $A I G a N / G a N-o n-s i l i c o n ~ S B D s$ with various FP designs.

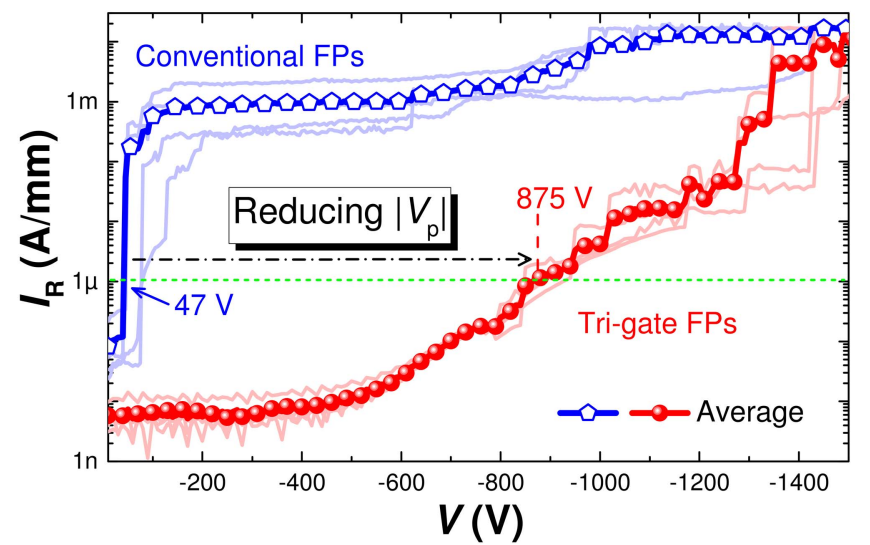

Fig. 5. Breakdown characteristics of the SBDs with and without the tri-gate FPs.

our model correlating small $I_{\mathrm{R}}$ with small $\left|V_{\mathrm{p}}\right|$, despite the different fabrication process and epi-layers.

One crucial benefit of the reduced $I_{\mathrm{R}}$ by decreasing the $\left|V_{\mathrm{p}}\right|$ is the enhanced soft $V_{\mathrm{BR}}$. Conventional FPs are usually based on oxide/AlGaN/GaN structures (Fig. 1(a)), which have large negative $V_{\mathrm{p}}$ due to the high carrier concentration in the 2DEG and charges in the oxide. This leads to a large $V_{\mathrm{SCH}}$, causing the $I_{\mathrm{R}}$ to increase rapidly to $1 \mu \mathrm{A} / \mathrm{mm}$ (at which the $V_{\mathrm{BR}}$ is typically defined), and resulting in a very small $V_{\mathrm{BR}}$. As the $\left|V_{\mathrm{p}}\right|$ is reduced, the leakage current through the Schottky junction saturates at smaller levels, until it is dominated by the highly resistive buffer layers at larger voltages, rather than only by the leaky Schottky junction. This leads to a significantly improved $V_{\mathrm{BR}}$, which is over $800 \mathrm{~V}$ in our case (Fig. 5).

\section{CONCLUSION}

In this work we presented a general approach to reduce the $I_{\mathrm{R}}$ in SBDs by reducing the $\left|V_{\mathrm{p}}\right|$ of their FPs, which led to ultra-low $I_{\mathrm{R}}$ below $10 \mathrm{nA} / \mathrm{mm}$ at $-500 \mathrm{~V}$ and enhanced the $V_{\mathrm{BR}}$ by over $800 \mathrm{~V}$. These results revealed the importance of a proper FP design for reducing the $I_{\mathrm{R}}$ in SBDs, unveiled the significant potential of the tri-gate FPs, and can potentially pave the path for efficient lateral SBDs for future power applications. 


\section{REFERENCES}

[1] J. Ma and E. Matioli, "High-voltage and low-leakage AlGaN/GaN tri-anode Schottky diodes with integrated tri-gate transistors," IEEE Electron Device Lett., vol. 38, no. 1, pp. 83-86, Jan. 2017, doi: 10.1109/LED.2016.2632044.

[2] C. W. Tsou, K. P. Wei, Y. W. Lian, and S. S. H. Hsu, "2.07-kV AlGaN/GaN Schottky barrier diodes on silicon with high Baliga's figure-of-merit," IEEE Electron Device Lett., vol. 37, no. 1, pp. 70-73, Jan. 2016, doi: 10.1109/LED.2015.2499267.

[3] Y.-W. Lian, Y.-S. Lin, J.-M. Yang, C.-H. Cheng, and S. S. H. Hsu, "AlGaN/GaN Schottky barrier diodes on silicon substrates with selective Si diffusion for low onset voltage and high reverse blocking," IEEE Electron Device Lett., vol. 34, no. 8, pp. 981-983, Aug. 2013, doi: 10.1109/LED.2013.2269475.

[4] M. Zhu, B. Song, M. Qi, Z. Hu, K. Nomoto, X. Yan, Y. Cao, W. Johnson, E. Kohn, D. Jena, and H. G. Xing, "1.9-kV AlGaN/GaN lateral Schottky barrier diodes on silicon," IEEE Electron Device Lett., vol. 36, no. 4, pp. 375-377, Apr. 2015, doi: 10.1109/LED.2015.2404309.

[5] J.-G. Lee, B.-R. Park, C.-H. Cho, K.-S. Seo, and H.-Y. Cha, "Low turn-on voltage AlGaN/GaN-on-Si rectifier with gated ohmic anode," IEEE Electron Device Lett., vol. 34, no. 2, pp. 214-216, Jan. 2013, doi: 10.1109/LED.2012.2235403.

[6] S. Lenci, B. De Jaeger, L. Carbonell, J. Hu, G. Mannaert, D. Wellekens, S. You, B. Bakeroot, and S. Decoutere, "Au-free AlGaN/GaN power diode on 8-in $\mathrm{Si}$ substrate with gated edge termination," IEEE Electron Device Lett., vol. 34, no. 8, pp. 1035-1037, Aug. 2013, doi: 10.1109/LED.2013.2267933.

[7] J. Hu, S. Stoffels, S. Lenci, B. De Jaeger, N. Ronchi, A. N. Tallarico, D. Wellekens, S. You, B. Bakeroot, G. Groeseneken, and S. Decoutere, "Statistical analysis of the impact of anode recess on the electrical characteristics of AlGaN/GaN Schottky diodes with gated edge termination," IEEE Trans. Electron Devices, vol. 63, no. 9, pp. 3451-3458, Jul. 2016, doi: 10.1109/TED.2016.2587103.

[8] J. Hu, S. Stoffels, S. Lenci, B. Bakeroot, B. De Jaeger, M. Van Hove, N. Ronchi, R. Venegas, H. Liang, M. Zhao, G. Groeseneken, and S. Decoutere, "Performance optimization of au-free lateral $\mathrm{AlGaN} / \mathrm{GaN}$ Schottky barrier diode with gated edge termination on 200-mm silicon substrate," IEEE Trans. Electron Devices, vol. 63, no. 3, pp. 997-1004, Jan. 2016, doi: 10.1109/TED.2016.2515566.

[9] E. Matioli, B. Lu, and T. Palacios, "Ultralow leakage current AlGaN/GaN Schottky diodes with 3-D anode structure," IEEE Trans. Electron Devices, vol. 60, no. 10, pp. 3365-3370, Oct. 2013, doi: 10.1109/TED.2013.2279120.

[10] J. Ma, G. Santoruvo, P. Tandon, and E. Matioli, "Enhanced electrical performance and heat dissipation in $\mathrm{AlGaN} / \mathrm{GaN}$ Schottky barrier diodes using hybrid tri-anode structure," IEEE Trans. Electron Devices, vol. 63, no. 9, pp. 3614-3619, Sep. 2016, doi: 10.1109/ TED.2016.2587801.

[11] B. Lu, D. Piedra, and T. Palacios, "GaN power electronics," in Proc. 8th Int. Conf. Adv. Semiconductor Devices Microsyst., Smolenice, Slovakia, Oct. 2010, pp. 105-110, doi: 10.1109/ASDAM.2010.5666311.

[12] S. Turuvekere, N. Karumuri, A. A. Rahman, A. Bhattacharya, A. Dasgupta, and N. DasGupta, "Gate leakage mechanisms in AlGaN/GaN and AlInN/GaN HEMTs: Comparison and modeling," IEEE Trans. Electron Devices, vol. 60, no. 10, pp. 3157-3165, Oct. 2013, doi: 10.1109/TED.2013.2272700.

[13] S. Oyama, T. Hashizume, and H. Hasegawa, "Mechanism of current leakage through metal $/ \mathrm{n}-\mathrm{GaN}$ interfaces," Appl. Surf. Sci., vol. 190, nos. 1-4, pp. 322-325, May 2002, doi: 10.1016/S01694332(01)00902-3.

[14] Y. Lei, J. Su, H.-Y. Wu, C.-H. Yang, and W.-F. Rao, "On the reverse leakage current of Schottky contacts on free-standing $\mathrm{GaN}$ at high reverse biases," Chin. Phys. B, vol. 26, no. 2, pp. 027105-1-027105-3, Dec. 2016, doi: 10.1088/1674-1056/26/2/027105.

[15] P. Pipinys and V. Lapeika, "Analysis of reverse-bias leakage current mechanisms in metal/GaN Schottky diodes," Adv. Condens. Matter. Phys., vol. 2010, Jul. 2010, Art. no. 526929. [Online]. Available: https://www.hindawi.com/journals/acmp/2010/526929/, doi: $10.1155 / 2010 / 526929$.
[16] Y. Lei, H. Lu, D. Cao, D. Chen, R. Zhang, and Y. Zheng, "Reverse leakage mechanism of Schottky barrier diode fabricated on homoepitaxial GaN," Solid-State Electron., vol. 82, pp. 63-66, Feb. 2013, doi: 10.1016/j.sse.2013.01.007.

[17] H. Zhang, E. J. Miller, and E. T. Yu, "Analysis of leakage current mechanisms in Schottky contacts to $\mathrm{GaN}$ and $\mathrm{Al}_{0.25} \mathrm{Ga}_{0.75} \mathrm{~N} / \mathrm{GaN}$ grown by molecular-beam epitaxy," J. Appl. Phys., vol. 99, pp. 023703-1-023703-6, Jan. 2006, doi: 10.1063/1.2159547.

[18] D. Yan, H. Lu, D. Cao, D. Chen, R. Zhang, and Y. Zheng, "On the reverse gate leakage current of $\mathrm{AlGaN} / \mathrm{GaN}$ high electron mobility transistors," Appl. Phys. Lett., vol. 97, no. 15, pp. 153503-1-153503-3, Jan. 2010, doi: 10.1063/1.3499364.

[19] S. Arulkumaran, T. Egawa, H. Ishikawa, and T. Jimbo, "Temperature dependence of gate-leakage current in $\mathrm{AlGaN} / \mathrm{GaN}$ high-electronmobility transistors," Appl. Phys. Lett., vol. 82, no. 18, pp. 3110-3112, Mar. 2003, doi: 10.1063/1.1571655.

[20] Z. H. Liu, G. I. Ng, H. Zhou, S. Arulkumaran, and Y. K. T. Maung, "Reduced surface leakage current and trapping effects in $\mathrm{AlGaN} / \mathrm{GaN}$ high electron mobility transistors on silicon with $\mathrm{SiN} / \mathrm{Al}_{2} \mathrm{O}_{3}$ passivation," Appl. Phys. Lett., vol. 98, pp. 113506-1-113506-3, Mar. 2011, doi: $10.1063 / 1.3567927$.

[21] S. K. Hong, K. H. Shim, and J. W. Yang, "Reduced gate leakage current in $\mathrm{AlGaN} / \mathrm{GaN}$ HEMT by oxygen passivation of $\mathrm{AlGaN}$ surface," Electron. Lett., vol. 44, no. 18, pp. 1091-1093, Aug. 2008, doi: 10.1049/el:20081350.

[22] E. J. Miller, D. M. Schaadt, P. Waltereit, C. Poblenz, J. S. Speck, and E. T. Yu, "Reverse-bias leakage current reduction in GaN Schottky diodes by electrochemical surface treatment," Appl. Phys. Lett., vol. 82, pp. 1293-1295, Feb. 2003, doi: 10.1063/1.1554484

[23] J. K. Sheu, M. L. Lee, and W. C. Lai, "Effect of low-temperaturegrown GaN cap layer on reduced leakage current of GaN Schottky diodes," Appl. Phys. Lett., vol. 86, pp. 052103-1-052103-3, Jan. 2005, doi: $10.1063 / 1.1861113$.

[24] J. Ma, X. Zhu, K. M. Wong, X. Zou, and K. M. Lau, "Improved GaN-based LED grown on silicon (111) substrates using stress/dislocation-engineered interlayers," J. Cryst. Growth, vol. 370, pp. 265-268, May 2013, doi: 10.1016/j.jcrysgro.2012.10.028.

[25] P. Drechsel, P. Stauss, W. Bergbauer, P. Rode, S. Fritze, A. Krost, T. Markurt, T. Schulz, M. Albrecht, H. Riechert, and U. Steegmüller, "Impact of buffer growth on crystalline quality of GaN grown on Si(111) substrates," Phys. Status Solidi A, vol. 209, no. 3, pp. 427-430, Jan. 2012, doi: 10.1002/pssa.201100477.

[26] J. Ma, Q. Zhuang, G. Chen, C. Huang, S. Li, H. Wang, and J. Kang, "Growth kinetic processes of AlN molecules on the Al-polar surface of AlN," J. Phys. Chem. A, vol. 114, pp. 9028-9033, Aug. 2010, doi: $10.1021 / \mathrm{jp} 100084 \mathrm{q}$

[27] R. Coffie, "Analytical field plate model for field effect transistors," IEEE Trans. Electron Devices, vol. 61, no. 3, pp. 878-883, Mar. 2014, doi: 10.1109/TED.2014.2300115.

[28] S. Karmalkar, M. S. Shur, G. Simin, and M. A. Khan, "Field-plate engineering for HFETs," IEEE Trans. Electron Devices, vol. 52, no. 12 , pp. 2534-2540, Dec. 2005, doi: 10.1109/TED.2005.859568.

[29] J. Ma and E. Matioli, "High performance tri-gate GaN power MOSHEMTs on silicon substrate," IEEE Electron Device Lett., vol. 38, no. 3, pp. 367-370, Mar. 2017, doi: 10.1109/LED.2017.2661755.

[30] S. Liu, Y. Cai, G. Gu, J. Wang, C. Zeng, W. Shi, Z. Feng, H. Qin, Z. Cheng, K. J. Cheng, and B. Zhang, "Enhancementmode operation of nanochannel array (NCA) AlGaN/GaN HEMTs," IEEE Electron Device Lett., vol. 33, no. 3, pp. 354-356, Jan. 2012, doi: 10.1109/LED.2011.2179003

[31] T. Boles, L. Xia, A. Hanson, A. Kaleta, and C. McLean, "Reverse leakage current and breakdown voltage improvements in GaN Schottky diodes," in Proc. Int. Conf. Compound Semiconductor Manuf. Technol. (CS MANTECH), Denver, CO, USA, May 2014 pp. 9-22. 